\title{
Urgences
}

\section{La poésie Oubedon}

\section{Gilles Marcotte}

Numéro 28, mai 1990

Le roman comme poétique

URI : https://id.erudit.org/iderudit/025590ar

DOI : https://doi.org/10.7202/025590ar

Aller au sommaire du numéro

Éditeur(s)

Urgences

ISSN

0226-9554 (imprimé)

1927-3924 (numérique)

Découvrir la revue

Citer cet article

Marcotte, G. (1990). La poésie Oubedon. Urgences, (28), 68-78.

https://doi.org/10.7202/025590ar d'utilisation que vous pouvez consulter en ligne.

https://apropos.erudit.org/fr/usagers/politique-dutilisation/ 


\section{La poésie Oubedon Gilles Marcotte}

C'est, bien entendu, à La luna de papel, haut lieu des échanges intellectuels, que Maryse rencontre le poète Adrien Oubedon. On n'est rendu qu'à la page soixante-neuf du roman, et la jeune fille n'est pas encore débarrassée de tous ses préjugés naïfs sur la poésie, bien qu'elle soit inscrite à la faculté de "littérologie». En voici un qui tombe: Adrien Oubedon, qui semble occuper dans le paysage poétique de l'époque un des premiers rangs, ne ressemble pas du tout à un poète. Il ressemble à tout le monde, il ressemble à n'importe qui:

Maryse avait toujours imaginé Oubedon sous les traits d'Émile Nelligan, mais il était gros et très quelconque. Ça ne faisait rien; il était tout de même poète, vivant, et assis en face d'elle. Sa figure un peu bouffie lui sembla mille fois plus agréable à regarder que celle de Lemire, qui lui faisait également face, et elle décida de centrer son attention sur le couple d'arrivants. ${ }^{1}$

Adrien Oubedon est poète comme d'autres sont bègues, mathématiciens ou conseillers municipaux. Il n'est même que cela, poète. Alors que les autres personnages du roman de Francine Noël ont une vie personnelle, des aventures personnelles, au-delà de leur définition professionnelle, Adrien Oubedon n'est jamais, dans Myriam premiere comme dans Maryse, qu'un fabricant de poèmes. Il n'y joue pas, d'autre part, un rôle de figurant, comme le professeur Surprenant, spécialiste de la "maudernité», avec qui Maryse a eu une prise de bec dans le chapitre précédent. Il insiste, il revient, toujours poète et rien que poète, représentant agréé de la fonction poétique jusque dans la cuisine de son ancienne muse Elvire - reconvertie en muse domestique dans Myriam première - où il fait irruption "avec ses gros sabots, sa besace et son gros ventre" $(M P, 178)$. La romancière d'ajouter : «Il est poète.»

1 Francine Noël, Maryse, Montréal, VLB éditeur, 1983, p. 69. Ce roman sera désormais représenté dans le texte par la lettre $M$. Le deuxième roman de Francine Noël, Myriam première (Montreal, VLB éditeur, 1987), sera représenté par les lettres MP. 
De ces quelques images, il est facile de conclure que la poésie ne constitue pas, dans l'œuvre de Francine Noël, un pôle d'attraction privilégié, une sorte d'idéal du langage comme celui qui investit par exemple le personnage de Steven dans l'œuvre de Victor-Lévy Beaulieu ${ }^{2}$, idéal dont le romancier lui-même, le prosateur, se sentirait exclu. Au contraire, la poésie, dans Maryse et dans Myriam premiere, fait partie de ces choses qui datent, qui viennent et s'en vont sans faire plus que rider la surface de lhistoire. La poésie, en somme, est un "look», comme la *littérologie", comme la «maudernité » du professeur Surprenant; et Adrien Oubedon ressemble à tout le monde, parce qu'il ressemble à son époque qui ne ressemble qu'à elle-même. Ainsi, dans Maryse, il est une sorte d'excroissance naturelle des années soixante: il est, comme Roland Giguère, un "homme de parole * $(M, 72)$; comme un poète de Parti pris, il proclame que "poésie et révolution sont les deux mamelles de l'intelligentsia ", que "la vraie vie est à l'usine» et que * la vraie voie passe par la Chine». Tout le monde sait, durant la Révolution tranquille, que "le métier de poète est aussi exigeant que celui de politicien, bien qu'il rapporte beaucoup moins *.

Le discours de Francine Noël sur la poésie semble donc être un discours contre la poésie, puisqu'elle la range parmi les effets de mode les plus fugitifs et ne lui reconnaît aucune forme possible de pérennité. Il parait, à cet égard, aussi radical, malgré ses apparences aimables, que celui de Réjean Ducharme sur l'évolution du goût dans Les enfantômes. On se souvient peut-être - mais ce n'est pas sûr, parce que cet admirable roman a été assez vite oblitéré par la critique que dans Les enfantômes Réjean Ducharme, par la voix de son personnage Vincent Falardeau, découpait le passé récent en décennies également décrites par les modes, le "look * : les "années carrantes " par leur voiture-fétiche, l'énorme De Soto, des actrices, Shirley Temple, Jeanne Crain, Loretta Young, des chansons, Does your chewing-gum loose its flavour on the bedpost overnight, Yes, we have no bananas, la querelle de l'« or figuratif» et le reste; puis les années cinquante; puis les années « soissantes", de la même façon, rejetant dans la même poubelle de l'histoire les expressions de l'art noble et celles de l'art commercial. Tout cela, scandé par le leitmotiv 
obsédant: « Je m'en souviens très bien *, formule incantatoire que la simple succession des événements et des modes ne cessait de contredire ${ }^{3}$. Cette atomisation de la mémoire, cependant, n'était pas un simple émiettement, une litanie d'insignifiances; elle était provoquée, comme une explosion, par la présence dans le récit de passions absolues, celle de Vincent Falardeau pour sa sœur Fériée et celle qu'ils entretenaient tous deux pour leur mère. Ce qu'on appelle le réel, la continuité du réel, n'avait aucune chance de tenir contre une telle puissance; il se décomposait, il s'émiettait. À certains égards, la succession des * look*, dans Maryse et Myriam première, obéit à une loi semblable, la mémoire familiale (la saga des O'Sullivan) jouant ici un rôle analogue à celui de la passion sororale et filiale dans Les enfantômes. Mais, parce que la relation œdipienne dans les romans de Francine Noël admet des médiations, des délais, des arrangements, elle rend pour ainsi dire habitable une temporalité pourtant aussi disjointe que celle des Enfantômes. La généalogie perd tout pouvoir, sans doute, dans ce monde aimable, mais les grands-mères deviennent des compagnes fort agréables, compréhensives, toujours jeunes de cœur, et les enfants, devant ces grands enfants que sont devenus leurs parents, exercent une indulgence souriante qui est du meilleur aloi.

Dans un tel monde, il va de soi que le poète Adrien Oubedon ne puisse apparaitre que comme daté, désuet par avance, borné par l'époque, l'actualité, victime des * tics et tics du temps qui passe. Il ne bénéficie même pas de l'immunité ethnologique qui appartient aux espèces en voie de disparition. Il reste assez présent, assez dangereux, aux yeux de la narration (qui est, elle, de quelle époque?) pour être mis par elle en accusation, sous trois chefs principaux:

3 La tendance * à chercher du sens dans des périodisations de plus en plus courtes ", dit Philippe Muray, avouerait en réalité le sentiment d'une absence de changement. « Les années 50, 80, Les Sixties, les Eighties. L'essence des décennies. Comme si on se doutait qu'il y a en réalité de moins en moins de différences possibles sous les chatoiements des changements de surface, qu'on est dans une sorte de grand trou qui dure, bien concret, qui s'éternise. Avec des événements bien sûr, et de multiples changements quand même. Pour que continuent les débats. Que les choses ajent l'air d'évoluer, de se modifier normalement." (Le $19^{\circ}$ siecle à travers les ages, Denöl, 1984, p. 34). Les choses changent tellement que rien ne change... 
1) Adrien Oubedon a le tort de se présenter comme un «poète professionnel» $(M P, 180)$, c'est-à-dire de prétendre mettre la poésie en coupe réglée, de lui assurer un statut officiel qu'elle ne saurait en aucune façon tolérer. À ce titre, il est plus coupable que le "logue ${ }^{4}$ - l'analyste, le théoricien, le professeur de littérature -, qui, lui du moins, n'a aucune prétention à la « création ". À La luna, dégonflant les discours idéologiques des copains, Adrien Oubedon, bien qu'un peu "gros », pouvait demeurer sympathique. C'est lorsque François et Maryse le découvrent en pleine activité de création que les choses se gâtent:

- Kriss-d'hostie-de-tabarnak-d'enfant-d'chienne-de-saintciboire-de-kaliss-de-kriss, dit-il, qu'est-ce qui vous prend de venir faire craquer mon plancher? J'vas débander ben raide, moi là!

- Wow, Adrien, dit François. Je viens seulement te porter les textes, pompe-toi pas.

- Tu 'aurais pu les laisser dans l'antichambre!

- Maudit que t'as donc mauvais caractère, toi! Heureusement que ça paraît pas dans ce que t'écris.

- J'ai pas mauvais caractère, je crée, c'est toute! Pis à part de t'ça, appelle-moi maitre comme tout le monde. $(M, 203)$

La caricature particulièrement appuyée de la dernière réplique donne à penser. Est-ce uniquement, principalement la professionnalisation de la poésie - on pensera à l'exaltation du poète dans l'aura de la Révolution tranquille et du mouvement indépendantiste - qui est visée ici? $\mathrm{Ne}$ serait-ce pas, plus outre, soulignée en caractères gras, l'idée même de la création, ou pour parler plus précisément, une idéologie de la création qui voudrait soustraire la poésie aux nécessités communes, à la parole de tous, à l'usage quotidien? Le poète (la poésie) serait ainsi fraudeur deux fois: en profitant professionnellement, politiquement de la poésie; mais surtout, plus gravement, en se donnant l'air d'echapper à l'usage honnête, responsable de la parole.

4 alle jubilait: Adrien Oubedon, dans l'intimité, était encore plus toffe à endurer que Michel! Elle eut le réflexe de remercier Dieu de vivre avec un logue plutót qu'avec un poète, mais elle se rappela à temps que Dieu était un grand carré beige. $"(M, 205)$ 
72

2) Ce n'est pas chez lui que Maryse et François vont déranger Adrien Oubedon - un poète a-t-il une demeure, a-til un lieu, une pierre où reposer sa pauvre tête? - , mais dans l'appartement de sa "muse" Elvire Légarée. Celle-ci prend son rôle très au sérieux, poursuit avec assiduité des études de «musologie» à l'université, et tient même bureau. Que fait une muse? Entrons, avec Maryse et François:

François et Maryse poussèrent la porte et restèrent sur le seuil, éblouis: Adrien, l'air extatique, actionnait la dactylo pendant qu'Elvire, immobile sur un monceau de coussins, dans la position du cobra à double menton, l'œil fixe et les bras mous, bruissait avec dévotion. (202)

Que veut dire exactement le verbe "bruire "? Le petit Robert nous indique qu'il vient du croisement de * rugir * et de "braire*, ce qui suggère un certain nombre de choses, mais ne saurait convenir entièrement à l'action ici décrite. Le sens, plus banal, de *rendre un son confus $*$, fera mieux l'affaire, puisqu'il s'accorde avec l'action d' « inspirer * dont il sera question plus loin. En somme, la * muse * ne fait rien; elle «inspire $"$, elle n'expire pas, ne produit pas; elle est toute dévotion, adoration. L'acte de créer est réservé au poète mâle qui, comme le dit plus ou moins volontairement l'aimable anglicisme du texte, * actionnait la dactylo». Une * dactylo* est une personne, selon le bon usage; et c'est aussi le rôle, le deuxième, qu'assume Elvire Légarée quand elle a fini d' * inspirer *, d'adorer, de «bruire». Elle copie, elle met au propre les textes du monsieur. Elle était, dans sa première fonction, confinée au pôle purement spirituel; elle n'est plus maintenant qu'une aide matérielle.

[...] le poème ne prend toute sa force, tout son sens, son suc, son tremblement, que lorsqu'il a été recopié en caractères standards par la muse. C'est une étape décisive dans le processus créateur car c'est alors seulement que le maître, voyant enfin son cuvre sortie de lui, extériorisée, peut la juger, la débarrasser de ses scories, l'émonder ou même la détruire.

Ainsi, qu'elle soit inspirante et inspiratrice ou servante - et le service consistera même à faire vivre Oubedon, "trop fragile pour la vie extérieure, trop sensible $(M, 272)-$, répudiable, échangeable à merci comme Elvire le découvrira bientôt, la "muse» est nécessaire au poète et exclue de la 
création poétique. Nous sommes loin de la prophétie de la Lettre du Voyant: "Quand sera brisé l'infini servage de la femme, quand elle vivra pour elle et par elle, l'homme, jusqu'ici abominable, - lui ayant donné son renvoi, elle sera poète, elle aussi! La femme trouvera de l'inconnu!» Adrien Oubedon, lui, réapparaissant dans Myriam premiere: «Voyant que Myriam écrit, il se penche amicalement vers elle. Il croit à la vocation des femmes-poètes - avant qu'elles n'atteignent l'âge de la puberté." $(M P, 179)$ Adrien le moderne, Adrien le poète-citoyen, ne fait en réalité que perpétuer l'asservissement de la femme, et tout donne à penser que ce n'est pas là un défaut de sa nature particulière, mais qu'il asservit la femme en tant qu'il est poète, parce qu'il est poète.

3) Le troisième chef d'accusation dressé contre Adrien Oubedon le taxe d'irresponsabilité, et il est formulé dans Myriam première, où le poète ne fait qu'une apparition, du reste substantielle, dans la maison de son ancienne "muse * Elvire Légarée reconvertie en muse domestique, fabricante de gâteaux qui l'ont rendue célèbre dans le voisinage. Elle a aussi trois enfants, dont deux au moins ont été faits avec la collaboration d'Oubedon. Mais celui-ci n'accepte d'en reconnaître qu'un seul, pour des motifs de pension alimentaire. Et d'autre part son amour pour le légitimé, le petit Hugo, reste chose de l'instant, passagère, sujette à de longues éclipses.

- Papa, papa! hurle un des enfants en tirant sur le gilet du poète, qui fond.

Littéralement, Oubedon se met à fondre: chaque fois qu'il s'entend appeler papa, il entre en transes. Pour quelques minutes. (181)

Restriction capitale: "Pour quelques minutes. . Et ce qui s'applique au fils vaut assurément pour la poésie, puisque Hugo (le nom qu'il a...), fils du poète et de la muse, est évidemment un poème, une figure de la poésie. Comme il n'est véritablement le père de Hugo que de temps à autre, Oubedon, bien que déclaré "poète professionnel», ne l'est qu'in partibus, à sa convenance, et fait penser au romancier américain de L'avalee des avalés, Blasey Blasey, qui bien qu'il écrive des romans extrêmement sexuels, revendique en dehors de l'exercice de son métier tous les honneurs dus à un mari et père de famille consciencieux. À vrai dire, parce qu'il est poète et parce que la poésie est naturellement plus excessive que le roman, Oubedon va plus loin, dans l'inconséquence et 
74

l'irresponsabilité, que son collègue prosateur de New York. Dans l'ordinaire même de l'existence, là où les libertés du poète devraient être limitées par les devoirs humains, il s'arroge le droit de retenir uniquement ce qui lui convient (Hugo) et de rejeter ce qui le gêne (Tristan, l'autre fils).

Irresponsable, Adrien Oubedon l'est encore en ce qu'il change de mode littéraire comme on change de chemise. Nous l'avions laissé, dans Maryse, « homme de parole « à la façon des années soixante, associant naturellement poésie et révolution, pénétré de l'importance du rôle politique du poète. Nous le retrouvons, dans Myriam première, complètement transformé (mais toujours poète, bien sûr: cela seul ne change pas): il a, comme le lui reproche Elvire, viré à droite; il parle des "écrivains post-Woodstock» $(M P, 183)$; il pratique la poésie "new wave (ibid.); en somme, il a le *beat des années 80 * $(M P, 181)$ et c'est pourquoi, comme tout le monde, il écrit un poème sur Montréal, intitulé «Ode à Maréal, ville mercantile et imaginative». S'il est encore vivant dans les années quatrevingt-dix - il le sera encore, les poètes du genre Oubedon sont increvables -, Adrien écrira ce qui doit s'écrire à cette époque, et c'est en cela qu'il est average, comme le dit Gabriel, frère aîné de Myriam: «Etant donné son âge, son background, son quotient intellectuel et son revenu annuel, Oubedon est typical. " Typique de quoi? De la poésie, voyons. Adrien Oubedon est celui qui gagne sur tous les tableaux, parce qu'il ne parie vraiment, il ne s'engage vraiment sur aucun: sa conception idéaliste de l'écriture lui interdit de dire en vérité le présent, de l'exprimer; et sa soumission aux modes invalide l'idéal même qu'il enclôt dans le mot "poète".

Pour l'essentiel, et bien qu'il emprunte quelques-uns de ses traits les plus acérés au féminisme contemporain, ce portrait-charge du poète n'est pas fondamentalement neuf: il y a belle lurette qu'on raille l'irresponsabilité du poète, ses prétentions un peu ridicules à l'action sociale, les petits ou grands profits qu'il tire de ses contorsions verbales, les sacrifices qu'il impose de gaieté de cœur à son entourage au nom de quelque mission plus ou moins sacrée. Si l'on veut faire montre d'une culture vraiment profonde, on remontera même jusqu'à Platon, qui traitait les poètes de menteurs - non sans raison, il faut le reconnaître - et proposait de les exclure de la cité. Mais l'intérêt du personnage d'Oubedon, souligné par la charge, la caricature à gros traits dont il est 
l'objet, vient plutôt de son inscription dans des circonstances historiques précises: il exprime le jugement porté par la littérature québécoise des années quatre-vingt sur la poésie des décennies précédentes ou, pour dire les choses autrement, sur la poésie même en tant qu'elle s'est manifestée, en tant qu'elle a agi dans ces décennies. À cet égard, il semble mais la question est plus complexe assurément - que Francine Noël prenne la position exactement contraire de celle d'un Victor-Lévy Beaulieu, prophète des années soixante-dix, qui dotait son personnage principal, le romancier Abel Beauchemin, d'une admiration et d'une envie presque maladives à l'endroit de son frère Steven, le poète. Pierre Nepveu a intitulé "Abel, Steven et la souveraine poésie " la belle étude qu'il a consacrée à cette rivalité 5 . Or, il est évident que la poésie ne saurait en aucune façon, pour Francine Noël, être "souveraine», et la romancière partage sur ce sujet la méfiance radicale d'un Jacques Ferron, pour qui le poète ne pouvait être qu'un fou ou un demi-fou, un exploiteur ou un imposteur (Saint-Denys Garneau au premier chef). Mais contre les prétentions indues de la poésie, Ferron ne faisait entendre que la protestation d'un individu; Francine Noël, au contraire, parce que son projet romanesque même l'y invite, porte sur la poésie le jugement d'une époque, celle des années quatre-vingt, qui serait non seulement "post-moderne" comme on le dit souvent, mais encore "postpoétique". Sans aller jusqu'à entériner simplement un tel jugement, contredit d'ailleurs par quelques œuvres majeures, on peut observer qu'en effet la poésie aujourd'hui, malgré la prolifération de ses titres - et peut-être même à cause de cette prolifération -, ne joue plus dans le paysage culturel québécois qu'un rôle secondaire, et n'oserait pas se présenter elle-même comme "souveraine *.

Mais on ne se débarrasse pas facilement du poétique et du poème, comme en fait foi le retour insistant d'Adrien Oubedon dans l'œuvre de Francine Noël; et surtout, il semble qu'on ne puisse le révoquer sans inscrire dans une autre forme l'essentiel de ses pouvoirs. Cette autre poésie, la trouveronsnous dans les quelques poèmes qu'écrit Maryse au cours de ses études en littérologie? Aucun signal d'ironie ne les accompagne. Au contraire, ils apparaissent dans le récit 
comme des moments de confidence nue, de pure vérité. Ils s'écrivent toujours au sein d'une crise ou après une crise, le plus souvent la nuit: le premier, *Poème triste* $(M, 124)$, alors que Michel a dû fuir le domicile commun à la suite des actions terroristes du FLQ; le deuxième $(M, 199)$ quand la narratrice relève à peine d'une grave maladie et se sent trahie par le même Michel; le troisième $(M, 398)$ après une rencontre malheureuse avec sa mère, à la suite de laquelle elle décide qu'elle n'aura jamais d'enfant. Le poème, pour Maryse, n'est toujours qu'une simple affirmation de vie pendant ou après l'épreuve, et ne s'embarrasse d'aucune ambition expressément créatrice, esthétique, sauf peut-être le deuxième qui d'ailleurs est le moins bon, le moins efficace, le moins touchant. La forme très coulante de vers libre qu'emploie Maryse, rappelant celle de la première génération de L'Hexagone, comfirme ce propos de simplicité, comme aussi la thématique du *Poème triste ", faite de l'opposition entre une suite de "Je ne suis pas... * qui exclut la narratrice de tout rôle prestigieux, et l'humilité très appuyée d'un "Je ne suis que...*:

Je ne suis ni la fille du roi,

Ni celle du juge Taschereau.

Je ne serai jamais la déesse rouge

de cette révolution avortée.

Je ne suis même pas politisée.

[...]

Je ne suis que la fille du pauvre Tommy O'Sullivan.

Je ne suis que la fille d'Irène Tremblay. (124)

La poésie est ainsi présente dans Maryse, mais en quelque sorte minimalisée, asservie à des nécessités particulières d'expression qui n'ont évidemment rien à voir avec quelque forme de « souveraineté * que ce soit. Le rythme poétique ne sert ici qu'à délivrer, à faire couler une plainte dans laquelle la narratrice se ressaisit, afin de passer à autre chose. Il arrivera même, dans le troisième poème, qu'aussitôt construit, le texte poétique soit biffé et remplacé, pour plus d'efficacité, par une phrase-affiche:

ON DEVRAIT POUVOIR CHOISIR SES AIIEULES. $(M, 398)$

Programme qui sera mis en œuvre, on le sait, par la romancière de Myriam premiere...

Cette poésie d'arrière-garde est sans doute pratiquée, dans Maryse, précisément parce qu'elle est d'arrière-garde, 
un peu démodée, et qu'elle n'a rien de commun avec les entreprises du genre Oubedon, qui revendiquent à la fois les privilèges spirituels de l'inspiration et la pertinence historique. Mais son innocence ne peut la préserver comme genre, comme discours spécifique, et c'est pourquoi elle est absente du deuxième roman de Francine Noël, Myriam première. Adrien Oubedon y revient, lui, mais pour être définitivement congédié, et avec lui toute forme d'ambition spécifiquement poétique. L'impérieuse Myriam, dont le jugement fait autorité en toutes matières principales et secondaires dans le roman qui porte son nom, affirme la supériorité de sa tante Maryse, femme de théâtre et bientôt romancière, sur les manœuvres douteuses du poète Oubedon:
Oubedon serait-il niaiseux, par hasard? Elle doute soudain qu'il soit un aussi bon écrivain que Maryse. Il ne doit sûrement pas être capable d'inventer des lettres d'amour, gros et tit-pickel [typical] comme il l'est. (MP, 180)

Mais la supériorité du roman sur la poésie n'est pas obtenue d'entrée de jeu, et à n'importe quel prix. La qualité essentielle de la poésie, dans l'univers de Francine Noël, est d'être facile (et l'on évoquera à juste titre le facile éluardien, qui a tant marqué la poésie québécoise de la Révolution tranquille); le roman, au contraire, s'écrit difficilement, longuement, sous le signe du travail. Mais un roman qui ne serait que travail, comme celui de François Ladouceur, remplirait-il les promesses de son genre?

Sur la porte fermée du bureau de François, on voit sa petite pancarte: Ne pas déranger, homme au travail. On entend le moteur de sa dactylo. Ce qu'il peut en dépenser, de l'énergie, son père, pour faire croire aux gens qu'il travaille quand il écrit ! [...] C'est pas comme madame Légarée: chez elle, ça coule de source, c'est de l'inspiré. François n'y croit pas, à l'inspiration, et c'est tant pis pour lui; il doit tout réécrire et ça lui prend du temps. (MP, 387)

Ne faudrait-il pas dire, à la fin, que la facilité seule (la poésie) et le travail seul (le roman) sont également inefficaces, improductifs? C'est le roman qui gagne - l'issue du combat était décidée depuis très longtemps -, mais dans la mesure où, par divers tours de passe-passe, il réussit à faire entrer dans son ordre, dans son large cours, les avantages de la poésie. Le roman, le vrai roman, sera celui qui, livré à la longueur du temps, au travail, sera en même temps traversé 
78

par les miracles de l'amour, du désir, de la fantaisie: «Elle [Maryse] aurait le goût d'écrire un long roman dans lequel tous les personnages seraient amoureux. Un roman de désir. * $(M P, 314)$ Ce roman, évidemment, c'est celui que nous avons sous les yeux déjà, que nous sommes en train de lire, où anges et "esprits mauvais" cohabitent aimablement avec les êtres humains dans une atmosphère de paradis terrestre à peine troublée par les drames du dehors, où grands-mères, mères et filles - avec un faux père par-ci par-là - s'entendent comme larronnes en foire pour déjouer les pièges de la lourde réalité.

L'œuvre romanesque de Francine Noël n'est pas seule, durant les années quatre-vingt, à voler son bien au poème, à pratiquer un dosage habile de travail romanesque et de légèreté poétique, de référence indiscutable et de gratuité verbale: qu'on pense, entre autres, aux récits de Jacques Poulin, de Sylvain Trudel, de Jacques Savoie. Ce ne sont pas des romans poétiques, au sens habituel de l'expression. Ils n'ont que faire de la poésie, parce qu'ils l'ont avalée. 